\title{
Face detection technique of Humanoid Robot NAO for application in robotic assistive therapy
}

\begin{abstract}
This paper proposed a face detection method for tracking the faces of children with Autism Spectrum Disorder in a robotic assistive therapy. The face detection is a novel approach in robotic assistive therapy involving autistic children since it is believe that those children will positively react with high-end devices, gadget and cutting edge devices. The intention of tracking the autistic children's faces is to measure the concentration level of the children in social interaction and communication since everyone knows that those children are suffering from communication disabilities and deficits due to brain developmental disorder. Humanoid Robot Nao with $573.2 \mathrm{~mm}$ height equipped with 2 internal cameras is utilized for this research. The face detection tools in choregraphe and telepathe based on Graphical User Interface (GUI) module is used in this study. The non-verbal interaction between humanoid robot and autistic children is recorded by using 2 internal cameras from the robot's head. The interaction is going to take about 30 minutes and supervised by occupational therapist and certified psychologist. The autistic children will be introduced to the Humanoid Robot Nao and their reaction will be recorded simultaneously while the robot is trying to track their faces.
\end{abstract}

Keyword: Autism spectrum disorder; Face detection; Humanoid robot; Robotic assistive therapy 